\title{
Computed Tomographic Assessment of Lateral Lamella of Cribriform Plate and Comparison of Depth of Olfactory Fossa
}

\author{
Bista $\mathrm{M},{ }^{1}$ Maharjan $\mathrm{M},{ }^{1}$ Kafle $\mathrm{P},{ }^{1}$ Shrestha $\mathrm{S},{ }^{1} \mathrm{KC} \mathrm{T}^{1}$ \\ ${ }^{1}$ Department of ENT and Head ad Neck Surgery, Kathmandu Medical College Teaching Hospital, Sinamangal, \\ Kathmandu, Nepal.
}

\section{ABSTRACT}

Introduction: Endoscopic sinus surgery is an upcoming branch in rhinological practice but with some major risks since it has to play around the skull base area. Lateral lamella of cribriform plate is the thinnest area of the skull base. Thus this study is undertaken to evaluate the height of lateral lamella of cribriform plate and the depth of olfactory fossae by the help of computed tomographic images.

Methods: Computed tomographic study of 50 patients was done in Advanced Imaging and Diagnostic center, Kathmandu Medical College. Coronal sections at the centre of infra-orbital foramina were taken as reference slide. The height of cribriform plate point was subtracted from the height of medial ethmoidal roof point to measure the length of lateral lamella of cribriform plates on both sides.

Results: The median height of LLCP in 100 slides was $2.8 \mathrm{~mm}$. LLCP height was 0 to $3.9 \mathrm{~mm}$ in 86 slides, 4 to $7 \mathrm{~mm}$ in 12 slides and greater than $7 \mathrm{~mm}$ in 2 slides. The LLCP length was greater in right side in $28(56 \%)$ patients and was greater in left side in $19(38 \%)$ patients. It was equal in both sides in only three patients $(6 \%)$.

Conclusions: As regards the length of LLCP; 0 to $3.9 \mathrm{~mm}$ length was most common. The olfactory fossa depth was more in the right side compared to the left side. Thus, right side is more vulnerable to injury during surgery. Thus adequate caution has to be exercised by the rhinological surgeon during endoscopic sinus surgery. 


\section{INTRODUCTION}

An endoscopic surgeon should have a clear idea of the anatomical variations around the anterior skull base area in order to prevent fatal iatrogenic complications. Lateral lamella of cribriform plate (LLCP) lies medial to fovea ethmoidalis, which forms the roof of the ethmoidal labyrinth - the orbital plate of the frontal bone. It separates the ethmoidal air cells from olfactory fossa. The LLCP is the thinnest anatomical structure in the skull base, offering lowest resistance to perforation during surgical maneuvers.

The depth of olfactory fossa is determined by the height of the lateral lamella of the cribriform plate. In 1962, Keros defined three heights and classified the depth of olfactory fossa in Keros type I (0 to $3 \mathrm{~mm}$ ), type II (4 to $7 \mathrm{~mm}$ ), type III (8 to $16 \mathrm{~mm}$ ). ${ }^{1}$

According to this classification Keros type III is the most dangerous because of the high chance of iatrogenic perforation during endoscopic sinus surgery. Besides this, ethmoid roof configuration tends to present lots of asymmetry in the height and angulation between the two sides even in the same individual. Here the bone is tenfold thinner than at the roof of the ethmoid. ${ }^{2}$ Advent of computer tomographic techniques has made preoperative CT a the road-map for sinus surgeries.

This study evaluates the frequency of different types of olfactory fossae among patients presenting to the hospital.

\section{METHODS}

An observational, hospital based prospective study was done in the department of ENT-HN surgery, Kathmandu Medical College Teaching Hospital, from January to June 2010. Ethical approval was taken from Kathmandu Medical College Ethical Committee. All the patients visiting ENT outpatient department with rhinosinusitis, nasal polyposis and headaches (facial pains) in the given period of time was taken. Total of 50 patients were included in the study group. The exclusion criterion were: patients under the age of 10 , previous history of surgery or trauma in the area of paranasal sinus or skull base area, congenital abnormalities of face and malignant diseases of the sinuses. Only coronal images were utilized in the study. The method for quantifying the position of the cribriform plate and ethmoid roof was relative to the orbital floor (as shown by the infraorbital nerve). This structure is easily identified on coronal CT scan. The plane of the slide for study went right through the infraorbital foramina (Figure 1,2,3). Two points were chosen as reference points at the skull base: the medial ethmoidal roof point (MERP), which corresponds to the medial ethmoidal roof at the point of articulation with lateral lamella of cribriform plate. The other point was the lowest point on cribriform plate (CP). ${ }^{3}$

The vertical height from the MERP to the horizontal plane, defined by the infraorbital nerve (termed as MERP height) and the vertical height from $\mathrm{CP}$ to the horizontal plane defined by the infraorbital nerve again (termed as CP height) was determined in each slide. The LLCP length was calculated by subtracting CP height from MERP height (MERP-CP $=$ LLCP). Comparative calculation of LLCP length in each slide according to Keros classification and comparision between the depths of olfactory fossae of both sides were calculated.

\section{RESULTS}

The CT scans of 50 patients were analyzed. Among them 28 were male and 22 were female. The youngest was 10 years old and the eldest was 78 years old. The median height of LLCP in 100 slides was $2.8 \mathrm{~mm}$. In this study Keros classification was used to categorize our data. The LLCP height was 0 to 3.9 in 86 slides (Figure 2). In 12 slides it was 4 to $7.0 \mathrm{~mm}$ (Figure 3). More than $7 \mathrm{~mm}$ was seen in only 2 slides (Figure 4). Analyzing the difference between the sides LLCP height was higher in the right side in 28 patients $(56 \%)$ and LLCP height was greater in left side in 19 patients (38\%). Only in 3 patients $(6 \%)$ the LLCP height of both sides were equal.

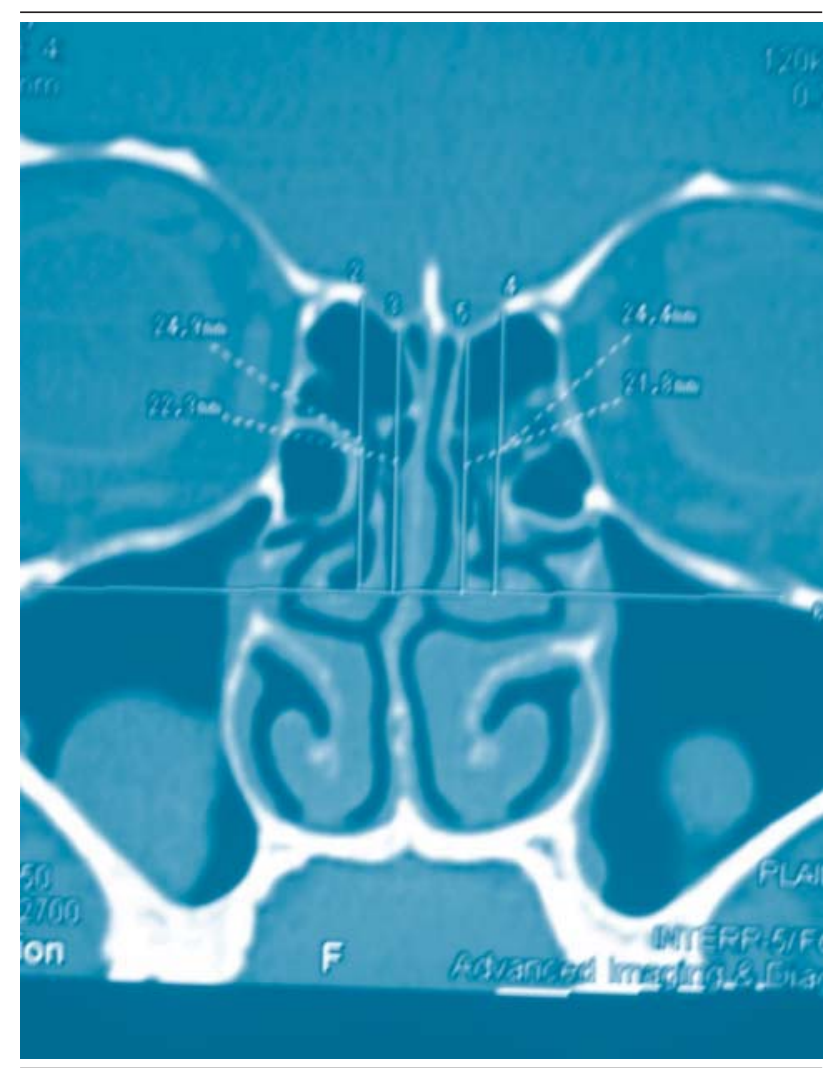

Figure 1. Keros type I. LLCP $=0$ to $(86$ sides in the study) 
Bista et al. Computed Tomographic Assessment of Lateral Lamella of Cribriform Plate and Comparison of Depth of Olfactory Fossae...

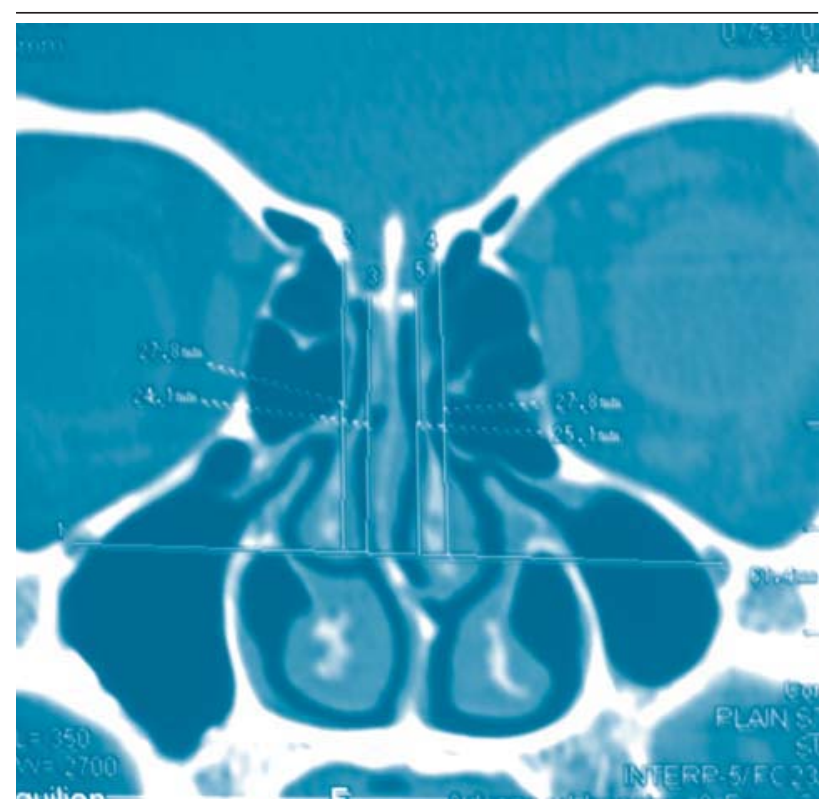

Figure 2. Keros type II. LLCP $=4$ to $7 \mathrm{~mm}(12$ sides in the study)

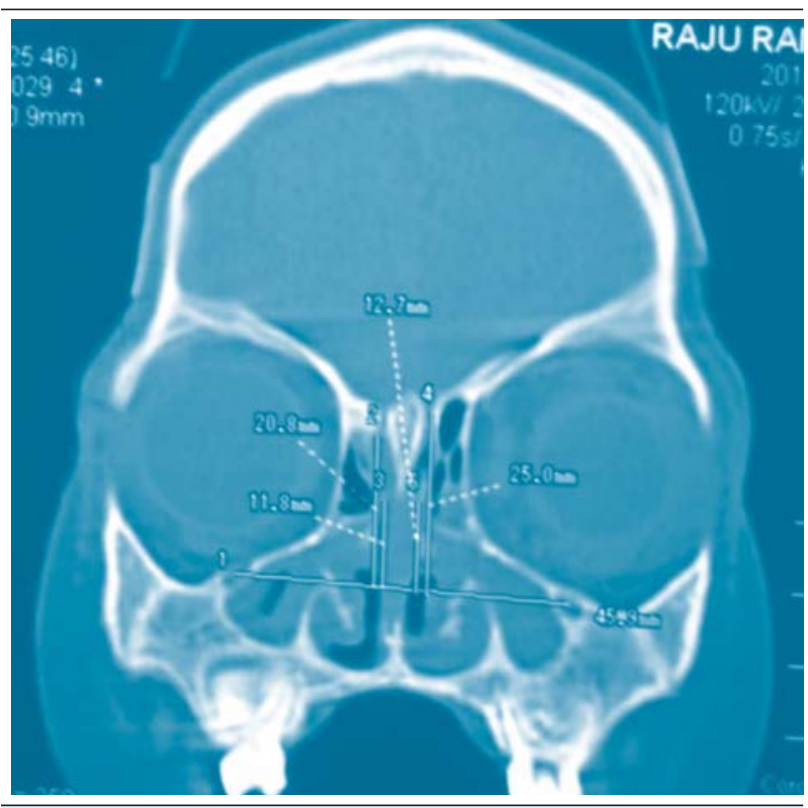

Figure 3. Keros type III. LLCP more than $7 \mathrm{~mm}(2$ sides in the study)

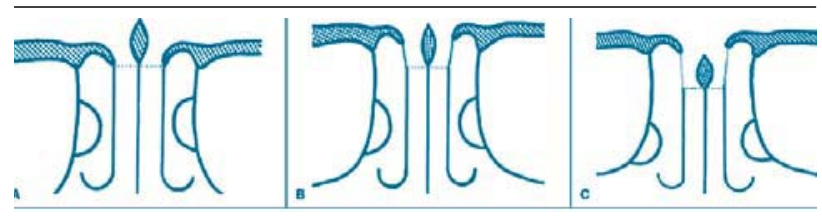

Figure 3. Figure 4. Schematic representation of the three types of olfactory fossae according to the Keros classification: A, type I; B, type II; C, type III. Courtesy - Radiologia Brasileira (print version 0100-3984)

\section{DISCUSSION}

The importance of evaluating the ethmoid roof by means of CT, for preventing iatrogenic complications has been explored by several studies in the past. Coronal plane is the best for evaluating ethmoid roof anatomy. ${ }^{4}$ It is well established that the area at risk is not in the highest point of ethmoid sinus formed by fovea ethmoidalis but in the lateral lamella of cribriform plate in the region of ethmoid sulcus. This is the most vulnerable site, in whole of the skull base. Here the anterior ethmoidal artery leaves the ethmoid sinus and comes anteriorly in the ethmod sulcus of the olfactory fossa. As mentioned earlier this study has been formulated according to Keros classification of ethmoid roof.

He proposed a 3- category classification based on height of LLCP in a study of 450 skulls. In the first type the olfactory groove was 0 to $3 \mathrm{~mm}$ deep and $12 \%$ specimen were of this type. ${ }^{1}$ In the current study all LLCP height of less than $4 \mathrm{~mm}$ were grouped together, this group had $86 \%$ of slides. Similar study by C. Arturo Solares et al showed $83 \%$ in this group in his study. The second type comprised all the cases in which LLCP height was 4 to $7 \mathrm{~mm}$ and $18 \%$ fell in this group in Keros specimen. In the current study this occurred in $12 \%$ cases. In the study by $\mathrm{C}$. Arturo Solares et al this group had 15 slides. ${ }^{4}$ Third type, where LLCP height was 8 to $16 \mathrm{~mm}$ was seen in $18 \%$ in Keros specimen. Where as in this study it was only $2 \%$. In the study by $\mathrm{C}$. Arturo Solares et al also had $2 \%$ only in this category. ${ }^{4}$ Basak et al,evaluated 64 children and found a high frequency of Keros type II (53\%) followed by type III (38\%)and least in type I (9\%) children. ${ }^{5}$ In the study by Soraia Ale Souza et al done in adult patients ,Keros type I was in $26.2 \%$ cases. Keros type II was seen in $73.3 \%$ of cases and Keros type III was seen in $0.5 \%$ of cases. ${ }^{4}$ The reason for the apparent differences in prevalence of the various LLCP heights are difficult to determine. It may be due to differences in technologies (cadaveric dissection Vs computer aided CT scanning).In some study the difference is seen due to the predominance of children group. Finally in this study and study by C. Arturo Solares et al, LLCP height of less than $4 \mathrm{~mm}$ were grouped as type I. This definition is more inclusive than type I definition of Keros were 0 to $3 \mathrm{~mm}$ was taken and were 3.1 to $3.9 \mathrm{~mm}$ is not mentioned.

The other important finding is the difference in the height of LLCP in right and left sides. As the height of LLCP increases the possibility of penetrating the skull base increases. Asymmetry in the right and left sides were seen in 47 out of 50 patients (94\%) in this study. Likewise the LLCP height was higher in the right side in 28 patients $(56 \%)$, this is also seen in the study by C. Arturo Solares et al. ${ }^{4}$ The LLCP height was seen higher in left side in 19 
Bista et al. Computed Tomographic Assessment of Lateral Lamella of Cribriform Plate and Comparison of Depth of Olfactory Fossae...

patients that is $38 \%$ of cases and equal in 3 patients $(6 \%)$. Wormald ${ }^{6}$, Dessi $\mathrm{P}$ et $\mathrm{al}^{7}$ and Lobowitz et $\mathrm{al}^{8}$ observed that the right fovea ethmoidalis was lower than the left side in more cases. Thus this study helps in navigation of the endoscopic procedure for safe surgery.

\section{CONCLUSIONS}

Most of the patient types I ( 0 to $4 \mathrm{~mm}$ ) variety of LLCP and the right olfactory fossa is deeper in more population than left. Therefore thorough evaluation of depth of olfactory fossa and presence of ethmoidal roof asymmetry in CT scans in the individuals elected for endoscopic nasal surgery is needed.

\section{ACKNOWLEDGEMENTS}

We acknowledge Advanced Imaging and Diagnostic Center (AIDC) located at Kathmandu Medical College. Their support and co-operation has immensely helped in completion of the study.

\section{REFERENCES}

1. Keros P. On the practical value of differences in the level of lamina cribrosa of the ethmoid. Z Laryngol Rhinol Otol. 1962;41:808-13.

2. Kainz J, Stammberger H. The roof of the anterior ethmoid: A Place of Lest Resistance in the Skull Base. Laryngol Rhinol Otol(stuttg). 1988;67:142-9.

3. Arturo SC, Lee WT, Batra PS, Citardi MJ. Lateral Lamella of the Cribriform plate. Arch Otolaryngol Head Neck Surg. 2008;134(3):285-9.

4. Souza SA, Souza MM Ale D, Idagawa M, Wolosker AMB, Ajzen SA. Computed tomography assessment of the ethmoid roof: a relevant region at risk in endoscopic sinus surgery. Radiol Bras. 2008;41(3)
5. Basak S, Akdilli A, Karaman CZ, et al. Assessment of some important anatomical variations and dangerous areas of the paranasal sinuses by computed tomography in children. Int J pediatr Otorhinolaryngol. 2000;55:81-9.

6. Wormald PJ. Surgery of the frontal recess and frontal sinus. Rhinology. 2005;43:82-5.

7. Dessi P, Moulin G, Triglia JM, et al. Difference in the height of the right and left ethmoidal roofs: a possible risk factor for ethmoidal surgery.Prospective study of 150 CT scans. J Laryngol Otol. 1994;108:261-2.

8. Lobowitz RA, Terk A, Jacobs JB, et al. Asymmetry of the ethmoid roof: analysis using coronal computed tomography. Laryngoscope. 2001;111:2122-4. 